\title{
Polydopamine / Cobalt Hexacyanoferrate Composite Films and Their Electrochemical Behavior in the Presence of Dpamine
}

\author{
Hanshuang ZHANG ${ }^{\mathrm{a},}{ }^{,}$, Lusheng $\mathrm{CHEN}^{\mathrm{b}}$, Xiuchong $\mathrm{LIN}^{\mathrm{c}}$ and Huaixiang $\mathrm{LI}^{\mathrm{d}}$ \\ College of Chemistry, Chemical Engineering and Materials Science, Collaborative Innovation Center \\ of Functionalized Probes for Chemical Imaging in Universities of Shandong, Key Laboratory of \\ Molecular and Nano Probes, Ministry of Education, Shandong Provincial Key Laboratory of Clean \\ Production of Fine Chemicals, Shandong Normal University, Jinan 250014, P. R. China \\ a756322383@qq.com , bchenlusheng2006@163.com, ${ }^{\mathrm{c}} 853103189 @$ qq.com, \\ dlihuaixiang@sdnu.edu.cn
}

\begin{abstract}
Polydopamine/cobalt hexacyanoferrate (PDA-CoHCF) composite films on a glassy carbon electrode (GCE) have been electropolymerized by using cyclic voltammetry. The composite films were electrochemically activated in a potassium hydroxide solution and were characterized by scanning electron microscope (SEM) measurements. The electrochemical activation could cause some changes of composite membrane interface structure and improve the electrochemical performance of the corresponding modified electrode in a phosphate buffer solution containing dopamine. The PDA-CoHCF film modified glassy carbon electrode could response dopamine sensitively in cyclic voltammetry measurements for electrochemical oxidation and reduction. The linear response slope and regression coefficient is $1.546 \mu \mathrm{A} / \mu \mathrm{M}$ and 0.985 , respectively, in the concentration range from $2.0 \mu \mathrm{M}$ to $10 \mu \mathrm{M}$ dopamine.
\end{abstract}

\section{Introduction}

The fabrication and broad applications of polydopamine (PDA) membranes have rapidly advanced in recent years and this trend clearly reveals the global significance of PDA and the intense interest of scientific research in this field [1]. The primary advantage of PDA is that, as seen with mussels, it can be easily deposited on wide range of inorganic and organic materials, including noble metals, oxides, polymers, semiconductors, and ceramics. Thus, PDA has opened a new route to the modification of various substrates and has stimulated extensive research. Because of its ease of use combined with its fascinating properties, PDA has attracted considerable interest for various types of applications, such as, PDA modified an anion exchange membrane to improve the selectivity of monovalent anions [2], using PDA as a free-radical scavenger to prepare a self-protected self-cleaning ultrafiltration membrane [3], improved mechanical properties and hydrophilicity of electrospun nanofiber membranes for filtration applications by PDA modification,

\footnotetext{
*Corresponding author:756322383@qq.com
} 
improving the electrochemical performance of silicon nanoparticle anode material by synergistic strategies of PDA and graphene oxide coatings, PDA microcapsules [4], etc. In the majority of these applications, the polymerization of dopamine is achieved via the chemical process that is easily implemented. A drawback of this method is that it is difficult to control the spatial localization and surface morphology of the deposited PDA thin film [5]. An alternative method for the preparation of PDA is through an electrochemical polymerization process. Since the electropolymerization only occurs in the vicinity of the electrode, PDA films can be generated in a highly controlled and spatially selective manne.

Among the metal hexacyanoferrates, cobalt hexacyanoferrate (CoHCF) would be especially attractive because of its excellent properties in electrocatalysis, capacity to store counter cations, and ion exchange selectivity. It exhibits excellent reversible redox centers very similar to those of Prussian blue while its electrochemical activity affected by electrolyte cation behaves differently from Prussian blue (PB) [6]. CoHCF has well defined and reproducible electrochemical responses because both the oxidized and reduced CoHCF structures seem to be fairly open and permit transport of alkali metal cations providing charge balance during redox reactions [7]. CoHCF does not undergo dissolution upon reduction or oxidation as the electrolyte ion diffuse in and out of the zeolitic structure of the compound to maintain charge neutrality. Thus, this material is an ideal candidate for composite with polymer, in especial with PDA.

Construction of PDA-CoHCF composite fimls based on electrochemical method has not yet been reported. According to the electrochemical activation principle, this study reports a new electrochemically polymerized PDA and $\mathrm{CoHCF}$ composite and then gradually activated by electrochemical method to cause the changes of composite membrane interface structure and the electrochemical behaviors. The composite membranes were characterized by scanning electron microscope (SEM) and cyclic voltametry (CV) measurements in the presence of DA phosphate buffer solution.

\section{Experimental Method}

Materials and Apparatus. Dopamine (DA) hydrochloride, ascorbic acid (AA) and uric acid (UA), potassium ferricyanide, $\mathrm{K}_{3} \mathrm{Fe}(\mathrm{CN})_{6} \cdot 3 \mathrm{H}_{2} \mathrm{O}, \mathrm{Co}\left(\mathrm{NO}_{3}\right)_{2}, \mathrm{Na}_{2} \mathrm{HPO}_{4} \cdot 12 \mathrm{H}_{2} \mathrm{O}$, $\mathrm{NaH}_{2} \mathrm{PO}_{4} \cdot 2 \mathrm{H}_{2} \mathrm{O}$, and other reagents were obtained from Sinopharm Chemical Reagent Co. Ltd. (Shanghai, China). All other reagents are of analytical reagent grade. All solutions were prepared with dissolved in doubly distilled water. A phosphate buffer solution (PBS) was made of $0.1 \mathrm{M} \mathrm{K}_{2} \mathrm{HPO}_{4}$ and $0.1 \mathrm{M} \mathrm{KH}_{2} \mathrm{PO}_{4}$ and adjusted to $\mathrm{pH}=7$ either using $\mathrm{KOH}$ $(0.1 \mathrm{M})$ or $\mathrm{HCl}(0.1 \mathrm{M})$. All cyclic voltametry $(\mathrm{CV})$ experiments were performed on a LK2005 electrochemical workstation (Lanlike Company, Tianjin, China). A practical three-electrode system was applied. The working, the auxiliary, and the reference electrodes were modified glassy carbon electrode (GCE, diameter: $3 \mathrm{~mm}$ ), platinum plate, and saturated calomel electrode (SCE), respectively.

Electropolymerization of Films. Prior to experiment, a GCE with the diameter of 3.0 $\mathrm{mm}$ was polished with diamond paste and ultrasonic cleaned sequentially in ethanol, acetone, $\mathrm{KOH}(1.0 \mathrm{M}), \mathrm{HNO}_{3}(0.1 \mathrm{M})$ and doubly distilled water. Polydopamine/cobalt hexacyanoferrate composite films were prepared by cycling the electrode potential between -0.10 and $+0.80 \mathrm{~V}$ (vs. SCE), $20 \mathrm{CV}$ scans at the scan rate of $50 \mathrm{mV} \cdot \mathrm{s}^{-1}$ in $0.1 \mathrm{~mol} \cdot \mathrm{L}^{-1} \mathrm{KCl}$ containing $1.0 \mathrm{mM} \mathrm{K}_{3}\left[\mathrm{Fe}(\mathrm{CN})_{6}\right], 2.0 \mathrm{M} \mathrm{DA}$ and $1.0 \mathrm{M} \mathrm{CoSO}_{4}$.

Activation of Films. The electrosynthesized Polydopamine/cobalt hexacyanoferrate composite (PDA-CoHCF) films were electrochemically activated by cycling the potential between -0.60 and $+0.80 \mathrm{~V}$ (vs. SCE), $5 \mathrm{CV}$ scans in $0.05 \mathrm{M} \mathrm{KOH}$ solution at the scan rate of $30 \mathrm{mV} \cdot \mathrm{s}^{-1}$ to stabilize the PDA-CoHCF) films.

Characterization of the Films. A glassy carbon plate electrode of surface area 1.0 
$\mathrm{cm}^{2}$ was utilized for elecrodeposition/electrodegradation and used for scanning electron microscope (SEM). TA FEI Sirion 200 SEM (Netherlands) was used to observe the surface morphology of the modified membranes.

Electrochemical Measurements. Standard solution of DA were prepared using doubly distilled water as solvent and stored under $281 \mathrm{~K}$. Electrochemical measurements of the composite film modified electrodes were carried out in $0.10 \mathrm{M}$ PBS containing a certain amount of DA. The CV cycles were realized between -0.6 and $0.6 \mathrm{~V}$ (vs. SCE), at a scan rate of $50 \mathrm{mV} \cdot \mathrm{s}^{-1}$. After each measurement, the modified films were removed and washed with water and scanned in blank PBS until the electrochemical signals of DA were disappeared.

\section{Results and Discussion}

Electropolymerization and Activation of PDA-CoHCF) Films. Cyclic voltametry was chosen to fabricate the composite films. Polymerization of polydopamine/cobalt hexacyanoferrate composite (PDA-CoHCF) was performed directly onto the GCE surface, which greatly simplified the fabrication procedure.

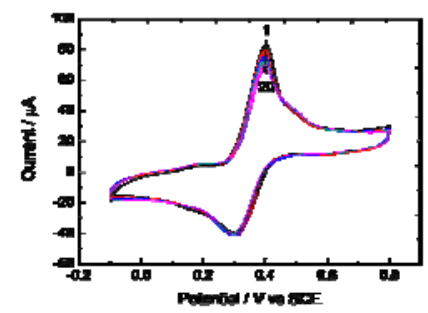

Fig. 1. Cyclic voltammograms for the electropolymerization of PDA-CoHCF in $0.1 \mathrm{M} \mathrm{KCl}$ containing $1.0 \mathrm{mM} \mathrm{K}_{3}\left[\mathrm{Fe}(\mathrm{CN})_{6}\right], 2.0 \mathrm{M} \mathrm{DA}$ and $1.0 \mathrm{M} \mathrm{CoSO}_{4}$. Scan rate: $50 \mathrm{mV} \cdot \mathrm{s}^{-1}$; number of scan cycles: 20 .

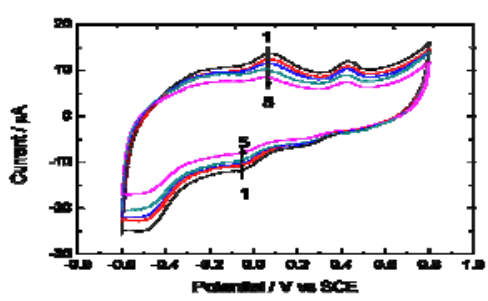

Fig. 2. Cyclic voltammograms for the electrochemical activation of PDA-CoHCF in $0.05 \mathrm{M}$ $\mathrm{KOH}$ solution on the GCE by cycling the potential between -0.60 and $+0.80 \mathrm{~V}$ (vs. SCE),. Scan rate: $30 \mathrm{mV} \cdot \mathrm{s}^{-1}$; number of scan cycles: 5 .

Fig. 1 shows the cyclic voltammograms recorded during the electropolymerization of PDA-CoHCF on the GCE at a scan rate of $50 \mathrm{mV} \cdot \mathrm{s}^{-1}, 20$ scan cycles. When the potential ranged from -0.10 to $0.80 \mathrm{~V}$, a sharp reversible oxidation peak appeared at $0.35 \mathrm{~V}$, which could be attributed to the formation of CoHCF. This oxidation peak current reduced gradually and stabilized when the completion of the 20 cycles. Corresponding reduction peak at $0.31 \mathrm{~V}$ keeps unchanged state indicating that the formed film is conductive and has redox behavior. A shoulder oxidation peak at about $0.48 \mathrm{~V}$ could be attributed to the formation of PDA, and corresponding the shoulder reduction peak at $0.15 \mathrm{~V}$. Thin films of PDA-CoHCF could grown electrochemically on GCE substrate either during a reduction process in a colloidal solution containing hexacyanoferrate(III), cobalt salt and potassium 
electrolyte [8]. Since in the oxidation and reduction process, dissolution and electrodeposition of films occurs alternately, so the deposited films are very compact and can adhere firmly to the electrode surface.

Fig. 2 shows the electrochemical activation process of the PDA-CoHCF film in a solution of potassium hydroxide. The activation process was performed by 5 cycles the potential between -0.60 and $0.80 \mathrm{~V}$ (vs. SCE) in $0.05 \mathrm{M} \mathrm{KOH}$ solution. As the increase of scans, both of the oxidation peak at about $+0.10 \mathrm{~V}$ and reduction peak at about $0.00 \mathrm{~V}$ decreased gradually, could be ascribed to some degradation of the composite film. This pair of redox peaks and other pair of redox at $0.45 \mathrm{~V}$ for oxidation and at $0.30 \mathrm{~V}$ for reduction suggest the composite film is provided with oxidation and reduction features since there is no redox species except $\mathrm{K}^{+}$and $\mathrm{OH}^{-}$in the solution, which nether is oxidized nor reduced at above mentioned potentials. Of course, the water could not be splitted.
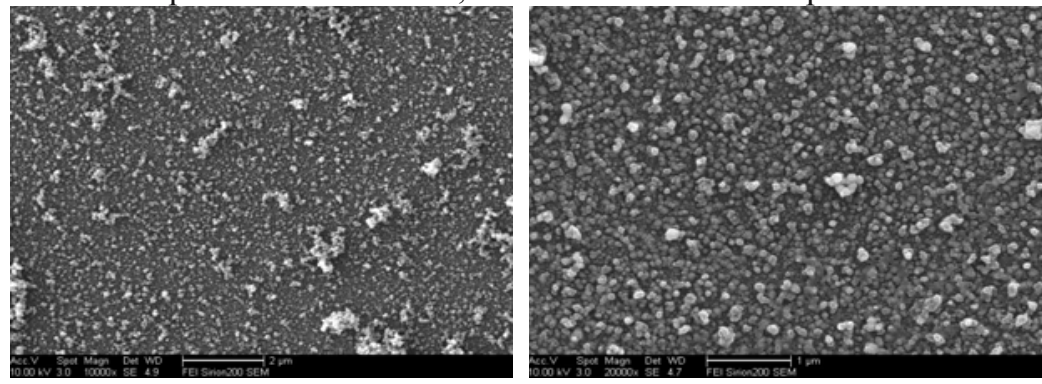

Fig. 3. SEM images of as prepared PDA-CoHCF (left) and after electrochemical activation in potassium hydroxide solution (right).

Fig. 3 shows scanning electronic microscope (SEM) images of as polymerized polydopamine/cobalt hexacyanoferrate composite film (left) and electrochemical activated polydopamine/cobalt hexacyanoferrate composite film on a glassy carbon electrode (right). We could see from the SEM images that the surface of GCE modified with as prepared polydopamine-cobalt hexacyanoferrate composite membrane is compact and random small spherical stockpiling, but after electrochemical activating, some particles became dilated and small apertures could be observed from the electrode surface. This indicated that the electrochemical activation could cause some changes of composite membrane interface structure and improve the electrochemical performance of the corresponding modifie electrode.

Electrochemical Behaviors of the Composite Films in PBS Containing Dopamine. The advantages of polydopamine-cobalt hexacyanoferrate composite membrane might arise from the adsorptive accumulation features for DA, which could be observed by the scan rate effects on the cyclic voltammograms. As can be seen in Fig. 4(A), where low concentration of DA $(2 \mu \mathrm{M})$ was used, obvious redox peaks were observed. There was no change in the peak potentials, but an increase of peak current was observed with the increasing scan rate in the range of $10-90 \mathrm{mV} \cdot \mathrm{s}^{-1}$.
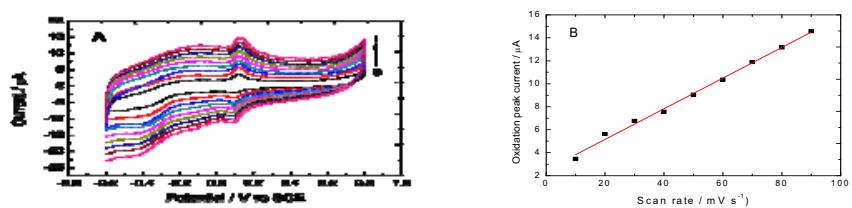

Fig. 4 (A) Effect of the scan rate on redox behaviour of DA in PBS (pH 7.4) on the PDA-CoHCF/GCE at various scan rates (a-i):10, 20, 30, 40, 50, 60, 70, 80, $90 \mathrm{mV} \cdot \mathrm{s}^{-1}$, respectively. The concentration of DA was $2 \mu \mathrm{M}$. (B) Oxidation peak current vs scan rate for the respective anodic peaks. 
The redox peaks were assigned to DA adsorbed on the PDA-CoHCF surface. This was further verified by the data analysis using plots of anodic peak current vs scan rate for the respective anodic peaks. Fig. 4(B) gives a linear equation: peak current $(\mu \mathrm{A})=2.484+$ 0.1345 scan rate $\left(\mathrm{mV} \cdot \mathrm{s}^{-1}\right)$ with a correction coefficient $\mathrm{r}=0.994$, indicating specific surface adsorption-controlled electron transfer mechanism for the respective redox process [9].

\section{Dopamine Concentration Response on the Modified Electrode}
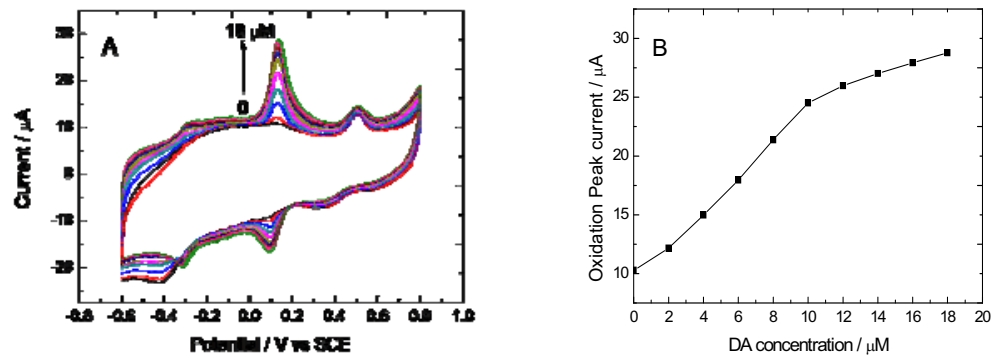

Fig. 5. (A) Cyclic voltammograms of PPABA/GCE $0.1 \mathrm{M} \mathrm{pH} 7 \mathrm{PBS}$ with different concentration of $0,2,4,6,8.10,12,14,16$ and $18 \mu \mathrm{M}$ DA at scan rate $50 \mathrm{mV} \cdot \mathrm{s}^{-1}$. (B) Plots of peak current vs. DA concentration

Because of the strong adsorption property of PDA-CoHCF film for DA, it was intended to develop a DA assay using PDA-CoHCF/GCE. Fig. 5(A) shows the well-defined CV oxidation peaks under optimized conditions. It is obvious that the currents increased with the increasing concentration of the DA standard solutions. Fig. 5(B) shows two the linear ranges, from 2 to $10 \mu \mathrm{M}$ with slope and regression coefficient of $1.546 \mu \mathrm{A} / \mu \mathrm{M}$ and 0.985 , respectively and from 10 to $18 \mu \mathrm{M}$ DA with slope and regression coefficient of 0.375 $\mu \mathrm{A} / \mu \mathrm{M}$ and 0.996 , respectively.. The detection limit is estimated to be $0.15 \mu \mathrm{M}(\mathrm{N} / \mathrm{S}=3)$ based on the first concentration range. This lower detection limit and higher sensitivities might be attributed to a porous composite polymer membrane on GCE, which would provide efficient detection of DA from the technological point of view.

\section{Summary}

A porous polydopamine-cobalt hexacyanoferrate composite film was formed by electrically polymerizing and then gradually electrochemically activated. Dopamine electrochemical oxidation peak current were linear responses. The porous polydopamine-cobalt hexacyanoferrate composite film modified glassy carbon electrode has a potential to fabricate a new type of electrochemical sensor for determination of DA.

\section{Acknowledgements}

This work was supported by the National Natural Science Foundation of China (No: 21405096; 21175084) and University Student Innovation Training Project of China (No: 20151517). 


\section{References}

1. Y. Liu, K. Ai, L. Lu, Polydopamine and its derivative materials: synthesis and promising applications in energy, environmental, and biomedical fields, Chem. Rev. 114 (2014) 5057-5115.

2. M. Vaselbehagh, H. Karkhanechi, R. Takagin, H. Matsuyama, Surface modification of an anion exchange membrane to improve the selectivity for monovalent anions in electrodialysis-experimental verification of theoretical predictions, J. Membr. Sci. 490 (2015) 301-310.

3. K. Feng, L. Hou, B. Tang, P. Wu, A self-protected self-cleaning ultrafiltration membrane by using polydopamine as a free-radical scavenger, J. Membr. Sci. 490 (2015) 120-128.

4. J. Shi, C. Yang, S. Zhang, X. Wang, Z. Jiang, W. Zhang, X. Song, Q. Ai, C. Tian, Polydopamine microcapsules with different wall structures prepared by a template-mediated method for enzyme immobilization, ACS Appl. Mater. Interfaces 5 (2013) 9991-9997.

5. Q. Liu, N. Wang, J. Caro, A. Huang, Bio-Inspired Polydopamine: A versatile and powerful platform for covalent synthesis of molecular sieve membranes, J. Am. Chem. Soc. 135 (2013) 17679-17682.

6. A. Malinauskas, R. Araminaite, G. Mickeviciute, R. Garjonyte, Evaluation of operational stability of Prussian blue- and cobalt hexacyanoferrate-based amperometric hydrogen peroxide sensors for biosensing application, Materials Science and Engineering C 24 (2004) 513-519.

7. S.J. Richard Prabakar, S. Sriman Narayanan, Amperometric determination of paracetamol by a surface modified cobalt hexacyanoferrate graphite wax composite electrode, Talanta 72 (2007) 1818-1827.

8. S.M. Chen, Characterization and electrocatalytic properties of cobalt hexacyanoferrate films, Electrochimica Acta 43 (1998) 3359-3369.

9. [9] J. Li, X. Lin, Simultaneous determination of dopamine and serotonin on gold nanocluster overoxidized polypyrrole composite modified glassy carbon electrode, Sens. Actuators B 124 (2007) 486-493. 